ESJ Humanities

\title{
Géomatique appliquée à la géomorphologie littorale : Contribution à la connaissance théorique des techniques d'analyse spatiale de l'évolution du linéaire côtier
}

\author{
Dr. Mamadou Thior
}

Laboratoire de Géomatique et d'Environnement (LGE), Departement de Géographie, Université Assane Seck de Ziguinchor, Sénégal

\section{Doi:10.19044/esj.2021.v17n28p101}

Submitted: 30 March 2021

Accepted: 10 July 2021

Published: 31 August 2021
Copyright 2021 Author(s)

Under Creative Commons BY-NC-ND

4.0 OPEN ACCESS

Cite As:

Thior M. (2021). Géomatique appliquée à la géomorphologie littorale : Contribution à la connaissance théorique des techniques d'analyse spatiale de l'évolution du linéaire côtier. European Scientific Journal, ESJ, 17 (28), 101. https://doi.org/10.19044/esj.2021.v17n28p101

\section{Résumé}

L'analyse de l'évolution du trait de côte en cinématique du littoral exige tout d'abord le choix d'un indicateur linéaire. En effet, il existe plus d'une douzaine de lignes de référence matérialisant la position du trait de côte. Cette diversité d'indicateurs induit la recherche et la mise au point de nombreuses méthodes pour détecter, extraire et suivre la mobilité du trait de côte. Ces approches méthodologiques reposent sur la compilation et la comparaison de données acquises, soit sur le terrain par des instruments de topométrie (niveau de chantier, théodolite, tachéomètre électronique, lidar, récepteur DGPS, etc.), soit en laboratoire par le traitement numérique d'images satellites ou aériennes. Le but de ce travail est de contribuer à une meilleure connaissance de l'approche cartographique et statistique qui permet de calculer les taux de variation historique du trait de côte à travers les outils la géomatique: Télédétéction et Système d'Information Géographique (SIG). La technique faisant l'objet d'étude dans cette approche est une méthode statistique d'extrapolation et de calcul de tendances basée sur le traitement des images géospatiales. Dans un cadre théorique, le sujet discute la définition de la ligne de référence, du protocole méthodologique de son extraction et de la cartographie de l'évolution du trait de côte du littoral. 
Mots clés : Trait de côte, Géomatique, Télédétéction, SIG, images géospatiales, la cartographie

\title{
Geomatics Applied to Coastal Geomorphology: Contribution to the Theoretical Knowledge of Spatial Analysis Techniques of the Evolution of the Coastal Line
}

\author{
Dr. Mamadou Thior \\ Laboratoire de Géomatique et d'Environnement (LGE), Departement de \\ Géographie, Université Assane Seck de Ziguinchor, Sénégal
}

\begin{abstract}
The analysis of coastline evolution in coastal kinematics requires first of all the choice of a linear indicator. Indeed, there are more than a dozen reference lines materializing the position of the coastline. This diversity of indicators leads to the research and development of numerous methods to detect, extract and monitor coastline mobility. These methodological approaches are based on the compilation and comparison of data acquired either in the field by topometry instruments (site level, theodolite, electronic tacheometer, lidar, DGPS receiver, etc.) or in the laboratory by digital processing of satellite or aerial images. The aim of this work is to contribute to a better knowledge of the cartographic and statistical approach that allows the calculation of historical coastline variation rates through geomatics tools: Remote sensing and GIS. The technique studied in this approach is a statistical method of extrapolation and calculation of trends based on the processing of geospatial images. In a theoretical framework, the subject discusses the definition of the reference line, the methodological protocol of its extraction and the mapping of the coastline evolution.
\end{abstract}

Keywords: Coastline, Geomatics, Remote sensing, GIS, geospatial images, mapping

\section{Introduction}

Par les expressions de Diaw (2003), les techniques de traitement et de gestion des données environnementales bénéficient depuis quelques décennies des moyens et des connaissances dynamiques où la télédétection et les systèmes d'information géographique fournissent une diversité d'outils d'investigation. La plupart des auteurs (Pennober, 2019 ; Byrnes, 1994 ; Boak et Turner, 2005 ; Faye, 2010) qui s’intéressent à la dynamique du littoral, en général, à la dynamique du trait de côte, en particulier, ont fréquemment mis 
en exergue toute la délicatesse de localiser et de positionner une limite entre la mer et la terre. A cette difficulté s'ajoute la diversité des critères d'identification du trait de côte selon les spécialités et la position des écoles. Limite de référence en tant que séparation linéaire entre deux domaines, terrestre et marin, le trait de côte est pourtant une notion ambiguë tant le passage de l'un vers l'autre est progressif dans l'espace et mobile dans le temps (Le Berre et al., 2008). Une synthèse de la littérature, établie par Robin et Gourmelon (2016), a ainsi mis en évidence plus d'une douzaine de limites de référence d'usage courant sur le littoral, dont plusieurs peuvent être considérées comme trait de côte.

Par ailleurs, la notion de trait de côte pose problème dès lors qu'elle change de signification littérale d'une langue à une autre. D'ailleurs, Bird (2007) soulignait déjà cette polémique conceptuelle. En effet, dans la littérature anglo-saxonne et américaine, en particulier, les termes shoreline et coastline sont considérés comme synonymes, alors qu'il faut les distinguer. Shoreline désigne la limite du plan d'eau en fonction des fluctuations du jet de rive et de la marée, ce qui signifie littéralement en langue française la ligne instantanée de rivage. Quant à l'expression coastline, elle renvoie à la limite atteinte par les pleines mers des vives eaux d'équinoxe et pouvant être matérialisée selon le type de côte par le pied de cordon ou limite de la végétation. Donc, en français, elle est liée à la notion de trait de côte.

Ainsi, l'extraction des repères peut être faite à partir des données de photographies aériennes, d'images satellites ou des documents cartographiques anciens. Considérés comme les principaux documents pour analyser l'évolution historique des littoraux, les documents historiques présentent initialement des sources d'erreurs de différentes natures. Idéalement, l'extraction de la position du rivage à partir de ces sources de données implique le géoréférencement et l'élimination des distorsions des cartes ou des photographies aériennes, suivie de la numérisation de la position du littoral (Morton et al., 2004; Hapke et al., 2016).

Evidemment, l'utilisation des documents cartographiques anciens par certains auteurs révèle des sources d'erreurs liées à leur fiabilité. La précision des levés topographiques et bathymétriques pose souvent des problèmes, car plus une carte est ancienne, moins elle est fiable. La précision de définition des niveaux de référence des marées n'est pas souvent précisée. L'utilisation des différentes cartes pourrait poser un problème si l'on ne s'assure pas que, dans toutes celles-ci, la nature de la marée est la même pour définir les marqueurs du trait de côte. Il est donc envisagé d'optimiser ces erreurs sur les niveaux de références des différentes cartes en s'inspirant des techniques de correction déjà réalisées dans la littérature existante.

De même que les photographies aériennes, les images satellitaires présentent des erreurs intrinsèques. Ainsi, Dolan et al., 1978 ont révélé sur les 
photographies aériennes des erreurs pouvant être liées aux changements d'altitude des satellites, déterminant des variations d'échelle d'une image à l'autre, les variations du relief entraînant une distorsion radiale et l'inclinaison de la caméra par rapport à la surface de la terre. Ces sources d'erreurs pourraient être contraignantes pour la fiabilité des résultats. C'est pourquoi l'harmonisation de l'échelle et la qualité des images aériennes sont essentielles pour une pertinente définition de la ligne de référence.

La représentation cartographique du trait de côte est très souvent symbolisée par une entité linéaire. Ainsi, il devient très difficile de percevoir les écarts spatiaux de deux traits de côte entre deux dates.

Cuq et al. (2002) ont souligné qu'il n'est pas facile de respecter des entités linéaires de manière à ce qu'elles soient clairement distinguées sur une carte, surtout quand la vitesse d'évolution n'est pas très rapide, car les positions successives d'un trait de côte à différentes dates sont traduites sur les documents cartographiques par un tracé d'une série de ligne géoréférencées pouvant, selon la situation, se confondre ou se croiser. Dans certains secteurs littoraux, évoluant à faible vitesse ou pour des suivis à haute fréquence temporelle, cette représentation n'est pas tout à fait satisfaisante, même si elle est utilisée à grande échelle. C'est pourquoi la difficulté à respecter les règles de sélectivité peut amener à une segmentation de la zone d'étude en cellules hydro sédimentaires. Ce choix peut être justifié par le fait qu'en raison de l'inadéquation entre une échelle trop petite (vaste zone) et une évolution du trait de côte peu marquée, on n'arrive pas à repérer visuellement une telle représentation. De ce fait, il est difficile de représenter la règle de sélectivité ou de séparation en cartographie du trait de côte.

Cependant, il arrive que la variation du trait de côte soit hétérogène. La réduction de l'échelle pour une représentation plus visuelle doit donc prendre en compte une situation de vue d'ensemble. C'est pourquoi la subdivision du littoral étudié en plusieurs segments est nécessaire. Par ailleurs, même si la représentation cartographique ne peut, à certains endroits, montrer clairement les écarts sur la disposition des différents traits de côte, la représentation graphique, elle, peut aussi montrer la cinématique. De ce fait, il est plus pertinent de combiner les deux types de représentation pour permettre une lecture aisée des résultats obtenus.

Il n'existe actuellement pas de norme largement acceptée pour l'analyse du changement des rives. Cette étude a donc pour but des rendre accessible un des aspects théoriques de la géomatique appliquée à la géomorphologie dynamique littorale. Pour cela, il est décrit dans cette contribution un protocole méthodologique efficace pour la détection et la mesure de la mobilité du linéaire côtier. 


\section{La ligne de référence: essai de définition de trait de côte}

La définition du trait de côte est délicate car son espace reste dynamique. Il s'agit tout d'abord de la ligne qui doit marquer la limite jusqu'à laquelle peuvent parvenir les eaux marines. On peut donc dire que c'est la limite la plus extrême que puissent atteindre les eaux marines, c'est-à-dire l'extrémité du jet de rive lors des fortes tempêtes survenues aux plus hautes mers de vives eaux. La position exacte au trait de côte mobile fait que plusieurs auteurs peuvent travailler sur le même thème, mais n'ayant pas la même définition. Leurs estimations sont probablement différentes. Dans la littérature existante, on retrouve des définitions de très grandes différences d'un auteur à un autre. Cela explique la différence entre Bourles (1998) et Boak et Turner (2005). En effet, pour Bourles, la limite est très mouvante d'autant plus qu'il s'agit de celle entre la côte et le rivage qui se déplace en fonction de l'état de la mer et de la marée. Il qualifie donc la marée de facteur déterminant du trait de côte.

Pour Boak et Turner (2005), on pourrait avoir une multitude de traits de côte; ce qui expliquerait d'ailleurs la possibilité pour eux de recenser 19 traits de côte à partir de 45 indicateurs relevés dans environ 80 publications. Le trait de côte n'a donc pas une définition, mais plusieurs, selon les indicateurs que les opérateurs lui attribuent pour l'identifier (Thior et al., 2019; Juigner 2012).

A côté de cette complexité qui revient constamment et est due à la mobilité des espaces d'estran, se posent les problèmes dus aux choix des différentes disciplines qui s'intéressent à la mobilité du trait de côte. Ainsi, en fonction de l'emplacement côtier, de la source de données et de la pertinence scientifique, différents indicateurs de position de la ligne de référence sont utilisés pour documenter les changements côtiers (Morton et al.,2004). Les lignes de référence peuvent varier donc d'une discipline à une autre. C'est pourquoi leur utilisation en cinématique du littoral a fait l'objet de plusieurs synthèses (Bird, 2007; Faye, 2010). Leurs différents travaux ont permis de ranger un ensemble de lignes de référence en 8 catégories selon les entités auxquelles elles se réfèrent. Mais, ce travail fait une synthèse de la littérature sur l'utilisation des différentes possibilités de lignes de référence.

\subsection{Critères d'utilisation de lignes de référence}

En fonction de l'objet d'étude, plusieurs critères /disciplines sont pris en compte: critères géomorphologiques, marégraphiques, botaniques, etc.

\subsubsection{Les critères géomorphologiques}

Les pieds de dunes simples. Dans la littérature anglaise, le pied de dune ou dune foot correspond à la limite supérieure de la haute plage. Le pied de dune est donc compris entre son front et la haute plage. Il peut être aussi 
localisé sur la dépression que l'on suppose être fréquemment sapée par les vagues. Cependant, plusieurs auteurs ont évoqué les limites de l'utilisation de cette entité linéaire comme ligne de référence. C'est ainsi que Stafford et Langfelder (1971); Faye (2010) notaient que le pied de dune est un bon indicateur d'érosion, mais les plages étudiées avec ce référentiel doivent connaitre pour la plupart du temps des phénomènes d'éboulement survenant de manière brusque.

Pied de dune végétalisé ; Il peut arriver que la végétation rampe jusqu'au pied de dune lorsque la pente de la dune est faible Dans ce cas de figure, on peut utiliser le pied de dune végétale comme marqueur du trait de côte. C'est d'ailleurs un cas qui permet de voir aussi bien la progradation que le recul. Cependant, lors des grandes marées de vives-eaux, la végétation rampante peut être engloutie du fait de la faible pente de la plage. L'instabilité de ce marqueur rend son utilisation difficile.

Les cordons raides et les ouvrages de protection car il peut arriver par endroits que la côte soit une falaise endossée; dans ce cas, le haut de la falaise et son pied reculent pratiquement au même rythme. En conséquence, l'un peut être considéré comme la ligne de référence. Aussi des unités géomorphologiques artificielles peuvent-elles naître suite aux constructions des murs de protection contre l'érosion côtière. Dans ce cas de figure, la base de l'infrastructure est la seule possibilité de marqueur de ligne de référence. Cependant, ces ouvrages réalisés pour lutter contre l'avancée de la mer ne permettent pas de faire une cinématique. De toute évidence, la ligne de rivage est ici fixée par l'ouvrage, ce qui ne permet pas de lire la mobilité «naturelle » du trait de côte. Et c'est pourquoi Boak et Turner, (2005) soutenaient qu'il n'est pas pertinent de faire une quelconque cinématique puisque les ouvrages de protection servent à fixer la ligne de rivage et peuvent être par moment modifiés par les hommes. Par conséquent, s'il doit y avoir une mobilité du trait de côte, elle ne sera pas naturelle. Cependant, les limites d'humectation issues des critères marégraphiques illustrent plus la mobilité naturelle du trait de côte.

\subsubsection{Les critères marégraphiques}

La marée est considérée comme ce mouvement périodique affectant les mers et qui se manifeste par une fluctuation de leurs niveaux. Les mouvements de la marée révèle, périodiquement, sur l'estran plusieurs positions susceptibles de faire l'objet de marqueur du trait de côte. Mais, il faut noter que la présence de cette diversité de repères fait que les critères marégraphiques sont moins fiables malgré la possibilité de corriger le niveau de marée. En effet, les lignes de référence sont détectées en fonction de la teneur en eau sur les plages à sable blanc. Or, les marées en période de vives eaux sont largement différentes de celles des périodes de mortes-eaux. Ce qui peut forcément être une source de confusion sur les différents indicateurs. 
C'est la raison pour laquelle les mouvements de la marée permettent d'avoir principalement comme ligne de référence la ligne instantanée de rivage, les limites d'humectation, la zone de saturation, les limites de sable mouillé/sec, les limites des pleines mers, limite des pleines mers habituelles.

La ligne instantanée de rivage où les plans représentés par la mer et le continent sont sécants selon une ligne qui est la ligne de rivage. Elle correspond donc à la surface linéaire marine sous l'influence de l'onde de marée et de la pente de l'estran à tout moment. Très mobile, la ligne instantanée de rivage est plus facile à remarquer sur les plages à large estran et à pente relativement faible. Mais, sur les côtes macrotidales, l'importance du marnage ne permet pas d'utiliser ce référentiel. La ligne supratidale, qui correspond à la limite de végétation dunaire, est alors le plus souvent utilisée. Aussi, la puissance du déferlement des vagues et le niveau de la marée peuvent-ils conditionner la position de la ligne instantanée de rivage. Ce qui fait que sur les littoraux à très fortes pentes, la ligne instantanée de rivage peut être confondue au pied de cordon dunaire. Stafford Langfelder (1971) pense que, même dans ce cas de figure, il n'est pas pertinent de parler de ligne instantanée de rivage, car le déferlement des vagues, qui traduit ce type de marqueur, peut être conditionné par les paramètres atmosphériques et météosmarins. Par conséquent, il peut y avoir une possibilité de déferlement précoce de la vague. Evidemment, quelle que soit la nature de la côte, la ligne instantanée de rivage y est présente. Néanmoins, le souci de ce marqueur est qu'il reste fluctuant d'une date à une autre et dépendant des conditions de marée. En plus, il est difficile de mentionner les informations sur les jours et les heures des prises de vue, et les hauteurs des marées correspondantes pour pouvoir effectuer les corrections nécessaires. Ces sources d'incertitude font que l'utilisation de la ligne instantanée de rivage est très peu fiable. Cependant, à court terme, l'utilisation de la ligne instantanée de rivage peut s'avérer relativement fiable, si un événement climatique majeur ne survient pas.

Les limites d'humectation à partir desquelles les lignes de niveau d'eau (lignes d'humectation d'estran) traduisent la limite supérieure du jet de rive, selon les différentes hauteurs du plan d'eau déterminées par les variations de la marée et les conditions des surcotes (Juigner et al., 2012). Ce sont des référentiels aisément détectables, notamment sur les images de haute résolution spatiale, mais elles doivent être caractérisées selon les conditions météo-marines précédant la date des relevés; ce qui n'est pas possible dans ce cas par manque de données pour les dates historiques. C'est pourquoi l'utilisation de la limite d'humectation est aussi contraignante que la ligne instantanée de rivage à cause de la complexité de l'espace côtier. En effet, il s'agit tout simplement des linéaires matérialisés par la limite du sable mouillé ${ }^{1}$

\footnotetext{
${ }^{1}$ Peut bien être sec, mais avec une avec une coloration assombris
} 
et du sable sec, surtout sur les plages à sable blanc où elles sont facilement observables.

La zone de saturation renvoie à la définition de Dolan et al., (1978) qui la considère comme l'extrémité du jet de rive à marée haute et, durant la marée descendante, migre vers la mer tout en marquant la limite côté terre des sables assombris par le déferlement. Son utilisation peut être pertinente sur les plages estuariennes à très basse altitude. En effet, à ce niveau, la plage sousmarine est de très faible pente et peut permettre un mouvement relativement régulier de ce type de marqueur du trait de côte. Mais toujours est-il que les conditions météo-marines, telles qu'expliquées plus haut, constituent un véritable élément qui perturbe la régularité de la limite d'humectation. Ici le nouveau paramètre qui perturbe est la variation diurne et par quinzaine de jours de la marée. Sur les côtes sénégalaises par exemple, la zone de saturation n'est pas au même niveau entre deux marées en une journée et en une quinzaine de jours. Or, il n'est pas souvent précisé sur les documents la période de marée haute au moment de la prise d'image. Par exemple, sur une image prise à marée haute, la zone de saturation est plus avancée que si elle devait être superposée à une autre image prise à marée basse. Ces mêmes paramètres changent également en fonction des périodes de vives et de mortes-eaux.

Les limites sable mouillé/ sable sec. Parmi les lignes de référence des limites d'humectation, peuvent être utilisés comme marqueur du trait de côte, les niveaux atteints par la mer sur l'estran. Mais, comme le nom l'indique, ce n'est plus le niveau de saturation qui les conditionne, c'est plutôt le niveau extrême que peuvent atteindre les déferlements. Son avantage est qu'il est parfois considéré comme équivalent à une ligne de pleines mers. Toutefois, comme toute limite d'humectation dépend des paramètres météo-marins, les limites entre le sable mouillé et le sable sec laissent voir logiquement des sources d'erreur citées précédemment. En plus de ces sources d'erreurs, son utilisation peut causer des problèmes liés cette fois-ci aux paramètres atmosphériques. Les limites sable mouillé/sable sec migrent à la fois en fonction de la marée et de l'insolation. Plus il y a du soleil et du vent, moins elles sont détectables. Par ailleurs, elles s'assèchent jusqu'à ce qu'elles se confondent avec les limites de la zone de saturation. La limite entre sable mouillé et sable sec est finalement comprise entre la zone de saturation et la limite des pleines mers.

Limite des pleines mers ou limites d'humectation migrent sur l'estran en fonction des conditions météo-marines et atmosphériques. Ainsi, lorsque les limites d'humectation atteignent leur plus haut niveau sur l'estran, on parle de limites des pleines mers. Elles correspondent selon Crowell et al., (1994), au niveau atteint par la dernière marée haute. En d'autres termes, elle correspond à la limite supérieure d'humectation de l'estran par la pleine mer précédente et elle est souvent matérialisée par le dépôt linéaire d'ordures. 
Plusieurs auteurs (Durand, 2004; Emmanuel, 2017) l'utilisent comme indicateur. En effet, son avantage est qu'il peut être un bon indicateur des mobilités saisonnières et d'identification des marées astronomiques. En plus, il est toujours présent même sur les plages à fortes pentes. Par ailleurs, les ordures linéaires de la plage qui la matérialisent font qu'elle n'est pas difficile à identifier, en comparaison avec les précédentes limites d'humectation. Cependant, comme les autres limites conditionnées par la marée, les limites de pleines mers présentent des contraintes liées à son utilisation. En effet, il peut arriver que les ordures ne soient pas existantes partout sur l'estran. Or la rupture matérielle (ordures) de ce type de marqueur pose donc un problème d'identification. De ce fait, son choix peut entraîner des erreurs d'estimation, si on ne parvient pas à la détecter au risque de la confondre avec la limite sable mouillé/sable sec ou encore le pied de dune.

Le pied de dune végétalisée ou la limite des pleines mers habituelles. Les limites d'humectation peuvent migrer jusqu'à atteindre les marqueurs de trait de côte de nature géomorphologique. On peut considérer ces limites comme des lignes exceptionnelles des eaux marines. C'est le cas des plages à très faibles pentes où la limite extrême d'humectation (pleines mers habituelles) est confondue avec le pied de dune. Il s'agit donc de la dernière limite influencée par les marées hautes des vives-eaux. Il présente les mêmes avantages que le pied de dune végétalisé. Cependant, il peut être une source d'erreur, si la précision n'est pas faite au préalable. Ainsi, dans la démarche, il est bien conseillé de préciser lequel des deux va comporter le choix du marqueur du trait de côte.

Les limites d'humectation dans leur globalité permettent un choix multiple de marqueur de trait de côte. En effet, dès qu'il y a une différence de teneur en eau sur les plages à sable blanc, se présente une possibilité de ligne de référence. C'est la raison pour laquelle elles présentent autant d'erreurs qui pourraient être liées à la confusion qu'elles donnent de la diversité de marqueurs du trait de côte. Par ailleurs, il faut noter que, dans cette étude, on ne précise pas réellement si les variations des positions des lignes de référence marégraphiques sont dues aux processus tectoniques, eustatiques ou aux deux à la fois, ou encore aux phénomènes isostatiques ou non. Mais, dans tous les cas, les variations des limites d'humectation sont considérées ici comme des mouvements relatifs apparents jusqu'à ce qu'elles soient nettement identifiées sur un support (Durand, 2004).

\subsubsection{Les critères botaniques}

Il s'agit des lignes de référence qui peuvent être matérialisées par la végétation. On peut avoir entre autres sur certains littoraux la limite inférieure de la pelouse dunaire, les limites de la végétation ligneuse, etc. 
Les limites de végétation sont utilisées en fonction des critères botaniques. Les limites de végétation peuvent être utilisées différemment aussi par rapport aux différents types de côtes. Dans de nombreuses circonstances, la limite de végétation est considérée comme un indicateur pratique pour diverses raisons. D'abord, elle est facilement identifiable au sol, sur l'imagerie satellitaire et aérienne; puis, elle coïncide généralement avec le bord de cordon, permettant l'utilisation d'un seul indicateur le long d'une côte; ensuite elle marque généralement le niveau d'eau extrême saisonnier le long des rivages aménagés; et c'est un excellent indicateur pour évaluer les retraits ou les avancées à long terme sur les rivages (Gould et al., 2015, Gibbs et al., 2017). On peut ainsi avoir, selon la limite inférieure de la pelouse dunaire, la végétation ligneuse.

La limite inférieure de la pelouse dunaire peut aussi être similaire au pied de dune végétalisé, surtout si la pente est relativement faible et que la végétation qui l'occupe est rampante. Elle présente aussi les mêmes sources d'erreurs que les limites citées plus haut avec les mêmes localisations. Il est donc essentiel de préciser les critères sur lesquels on se base pour caler la ligne de référence. Ce type de marqueur du trait de côte a l'avantage de montrer à la fois une progradation momentanée et un recul, puisque la végétation peut ramper ou reculer en fonction de la mobilité de la dune qui, elle aussi, est dépendante du transit sédimentaire sur la plage.

Les limites de la végétation ligneuse désignent la végétation pérenne qui ne peut se développer que sur l'arrière-plage, c'est-à-dire sur les dunes à fort escarpement. A ce niveau, les limites de végétation peuvent être détectées sur les fronts dunaires. Ainsi, si la pente de la dune est raide (abrupte), les limites de végétation reculent au même rythme que les pieds de dune normale et, par conséquent, ils auront les mêmes estimations. Ce type de marqueur est bien adapté sur les littoraux à végétation artificielle. On peut les rencontrer sur les dunes de certaines plages de la végétation, qui a été plantée pour fixer la dune, constituent un bon indicateur d'érosion. En revanche, il faut noter que les limites de la végétation artificielle ne sont pas des indicateurs d'évolution naturelle du trait de côte. Plus la limite est naturelle, plus elle répond aux critères initialement prévus. Or, les plantations de filaos par exemple ont pour but de ralentir l'avancée de la mer. Ce qui fausserait naturellement l'estimation de la mobilité du trait de côte. En plus, ce type d'indicateur ne montre pas forcément une progradation naturelle.

Ces lignes de références sont les plus fréquemment utilisées sur les côtes meubles. Toutefois, il est conseillé de faire preuve de prudence en utilisant la ligne de végétation comme indicateur du littoral autour des centres très urbanisés, où sa position peut être significativement modifiée par des perturbations humaines (Gould et al., 2015, Gibbs et al., 2017). Dans tous les cas, les limites de la végétation, qu'elle soit ligneuse ou encore de la pelouse 
dunaire, sont partout remarquables sur les côtes végétalisées. Il existe, cependant, d'autres types de marqueurs du trait de côte faisant partie de la douzaine non expliqués. On peut citer entre autres les lignes de référence virtuelles, l'étendue de plage.

\subsubsection{Les lignes de référence virtuelles}

Elles échappent à une vision physique et ne peuvent être détectées que de manière optique. C'est pourquoi le traitement des images montre comme ligne de référence des comportements spectraux linéaires (Boak et Turner, 2005). Mais, du fait de leur existence éphémère ${ }^{2}$, l'utilisation des lignes de référence virtuelle est un risque pour la fiabilité des informations.

\subsubsection{L'étendue de la plage}

Elle fait l'objet de débats entre plusieurs auteurs sur sa délimitation exacte, puisqu'il s'agit d'une délimitation de l'estran. En effet, la limite supérieure peut rester intacte, car constituant en amont le pied de dune ou la limite de la végétation, s'il s'agit d'un abrupt. Mais, le souci de la délimitation de l'étendue de la plage reste sa limite inférieure. Ainsi, entre la limite des pleines mers, la limite de sable mouillé/sable sec, et la ligne instantanée de rivage, laquelle doit être considérée comme limite aval de l'étendue de plage ? On peut dire que les limites d'humectation reviennent à nouveau comme choix, mais cette fois pour marquer la limite aval de l'étendue de la plage. Par conséquent, l'étendue de la plage peut être très limitée pour mesurer l'évolution linéaire du trait de côte. En revanche, elle est bien utilisée dans certaines études spatiales pour estimer les pertes surfaciques de terrain induites par l'érosion côtière.

On peut donc retenir qu'il existe plus d'une douzaine de lignes de référence pour marquer un trait de côte. Ainsi, selon les différents critères, les lignes de référence peuvent être identifiées en fonction du type de plage. A l'évidence, il se pose des contraintes liées tant à leur utilisation qu'aux résultats escomptés. Les données utilisées en cinématique, étant très souvent les images et les photos aériennes, les erreurs radiométriques et géométriques devront minutieusement être corrigées, surtout pour les calculs des taux de recul du trait de côte. C'est pourquoi il est nécessaire de bien préciser la démarche adoptée pendant l'extraction des lignes de référence utilisées.

\subsection{L'extraction de lignes de références}

L'extraction des marqueurs de trait de côte peut se faire manuellement sur les données géospatiales. Ces marqueurs de trait de côte peuvent directement être visibles sur l'image, si celle-ci a une bonne résolution. C'est

${ }^{2}$ Il s'agit des lignes instantanées de rivage qui ne durent pas plus d'une dizaine de minutes 
la raison pour laquelle la photo-interprétation permet d'extraire manuellement les lignes de référence ou bien elle est assistée par ordinateur. Précisons que les marqueurs les plus faciles à détecter manuellement sont les lignes instantanées de rivage, les limites d'humectation et les limites de végétation. En revanche, le trait de côte le plus souvent utilisé par les chercheurs est la ligne de rivage. C'est pourquoi, Dolan et al. (1978) soulignent le consensus qui est fait sur le niveau de marée haute, puisqu'il est continu le long du littoral, facilement reconnaissable sur les photos, car il est situé à la limite entre les parties mouillées (qui apparaissent en gris foncé sur les photos) et sèches (apparaissant en gris clair à blanc) de la plage, enfin parce qu'il varie le moins horizontalement en fonction de la marée.

\subsubsection{L'extraction de lignes de références : exemple sur les images de Landsat}

L'analyse spatiale se fait alors sur la base d'une combinaison de la télédétection et des Systèmes d'Information Géographique (SIG). Ainsi, l'image utilisée ici est celle de Landsat 5 du 09-02-1986 du capteur TM pour servir d'exemple.

Les lignes instantanées de rivage et les limites d'humectation sur les images Landsat. La faible résolution des images satellitaires de Landsat 5 (30 $\mathrm{m})$ ne permet pas une bonne reconnaissance d'une ligne sur l'étendue de plage. Mais elles peuvent subir des traitements sur les côtes caractérisées par la présence quasi totale d'une végétation côtière qui peut border la plage. Alors, au passage du satellite, certains indicateurs retrouvés sur l'étendue de plage (ligne de rivage, limites d'humectation) sont pratiquement inexistants et la résolution spatiale des images ne permet pas de faire une distinction entre la ligne instantanée de rivage et celle des pleines mers des eaux précédentes qui font partie des limites d'humectation. Faye (2010) signalait qu'au sud de la presqu'île du Cap-Vert, en plus de la ligne instantanée de rivage, la limite supérieure de la plage peut être plus ou moins distinguée sur tout le littoral jusqu'au Rio Cacheu et, ponctuellement, sur les segments de côtes sableuses au sud de ce secteur. Cependant, il est plus prudent d'éviter ces types d'indicateurs sur les images de Landsat s'il apparaît que la limite supérieure de la plage peut être matérialisée sur le front de mer par les contours de tapis végétal. Néanmoins, les limites de la végétation sont facilement détectables sur les images satellitaires de Landsat.

Les limites de la végétation sur les images Landsat où l'indicateur sélectionné sur les images satellitaires de Landsat est naturellement la limite de la végétation. L'intérêt accordé à ce référentiel sur ce type de données est le plus défendu par plusieurs auteurs (Faye, 2010; Bird, 2007; Morton et al., 2004); sa précision sur la nature de la côte, permet de le distinguer. Ils considèrent que la limite de la végétation en front de l'étendue de plage (du 
côté de la mer), qu'elle soit dunaire (Vegetation line, seaward edge of dune vegetation) ou encore pérenne (Line of permanent, stable, long-term vegetation), est une entité bien visible sur les images Landsat, si une composition colorée correspondante y est appliquée. A cet effet, les côtes végétalisées présentent donc des caractéristiques favorables à l'utilisation de la limite de la végétation comme ligne de référence. En revanche, il est important de signaler que les limites du côté de la mer de la végétation ligneuse ou pérenne et celle de la pelouse du tapis végétal n'ont pas les mêmes rythmes de cinématique. Evidemment, la limite de la végétation ligneuse ou pérenne recule le plus souvent et ne progresse que lorsqu'une sédimentation est assez importante et qu'elle perdure sans connaître d'érosion d'origine marine. Dans certains cas, le front de la limite de la végétation ligneuse peut progresser suite à un reboisement artificiel. En revanche, les pelouses des tapis végétaux sont bien utilisées pour caractériser une évolution du trait de côte marquée aussi bien par une situation de progradation qu'une situation d'érosion ou encore une situation de stabilisation. Cela s'explique par le fait que cette végétation, très souvent rampante, a la capacité de pousser sur des accumulations récentes. De ce fait, il apparaît plus facile d'observer une évolution marquée par les trois situations que si on utilise la limite de la végétation ligneuse. C'est la raison pour laquelle, sur les images satellitaires de Landsat, ces deux types de végétation ont donc des réflectances très proches, et une bonne connaissance du terrain est nécessaire pour les distinguer.

\subsubsection{Le traitement des images satellitaires Landsat}

Du visible à l'infrarouge, plusieurs images sont fournies par le capteur TM. Il s'agit donc ici d'adopter une démarche qui accorde plus d'importance aux informations ciblées sur le terrain. Étant donné que la végétation dans ce choix est incontournable pour l'extraction de la limite de la végétation, le canal rouge du spectre électromagnétique demeure adéquat pour le traitement, d'autant plus qu'il fait mieux ressortir le contact entre les sédiments secs de la haute plage et la couverture végétale (Robin et Gourmelon, 2016). De ce fait, une composition colorée et une classification supervisée demeurent nécessaires pour regrouper les zones à comportements spectraux proches ou similaires. Barry (2016), utilisant ce même référentiel sur toute la côte de Kafountine sur toutes les dates étudiées, a effectué des compositions colorées qui sont obtenues à partir d'une superposition de trois bandes spectrales sur trois niveaux de couleurs primaires, soit le Rouge, le Vert, et le Bleu (RVB). Par ailleurs, dans la plupart des études de télédétection sur la végétation, ce type de composition colorée est très souvent utilisé, car la végétation a une forte réflectance dans le proche infrarouge au moment où l'eau présente une faible réflectance dans le domaine infrarouge du spectre électromagnétique (Girard, 2010). Ensuite, un seuillage a permis d'identifier, grâce à une limite 
nette (ligne de référence), les populations de pixels représentant la zone terrestre (pixels clairs) et le domaine marin, c'est-à-dire des pixels sombres (Djagouna, 2016). Ainsi, pour la composition colorée de l'image de 1986, la combinaison des bandes 3, 21 ou 4, 3, 2 donne une bonne couleur à la végétation sur l'image (fig.1). Aussi les bandes 3, 2, 1 donnent-elles une couleur naturelle à l'image.
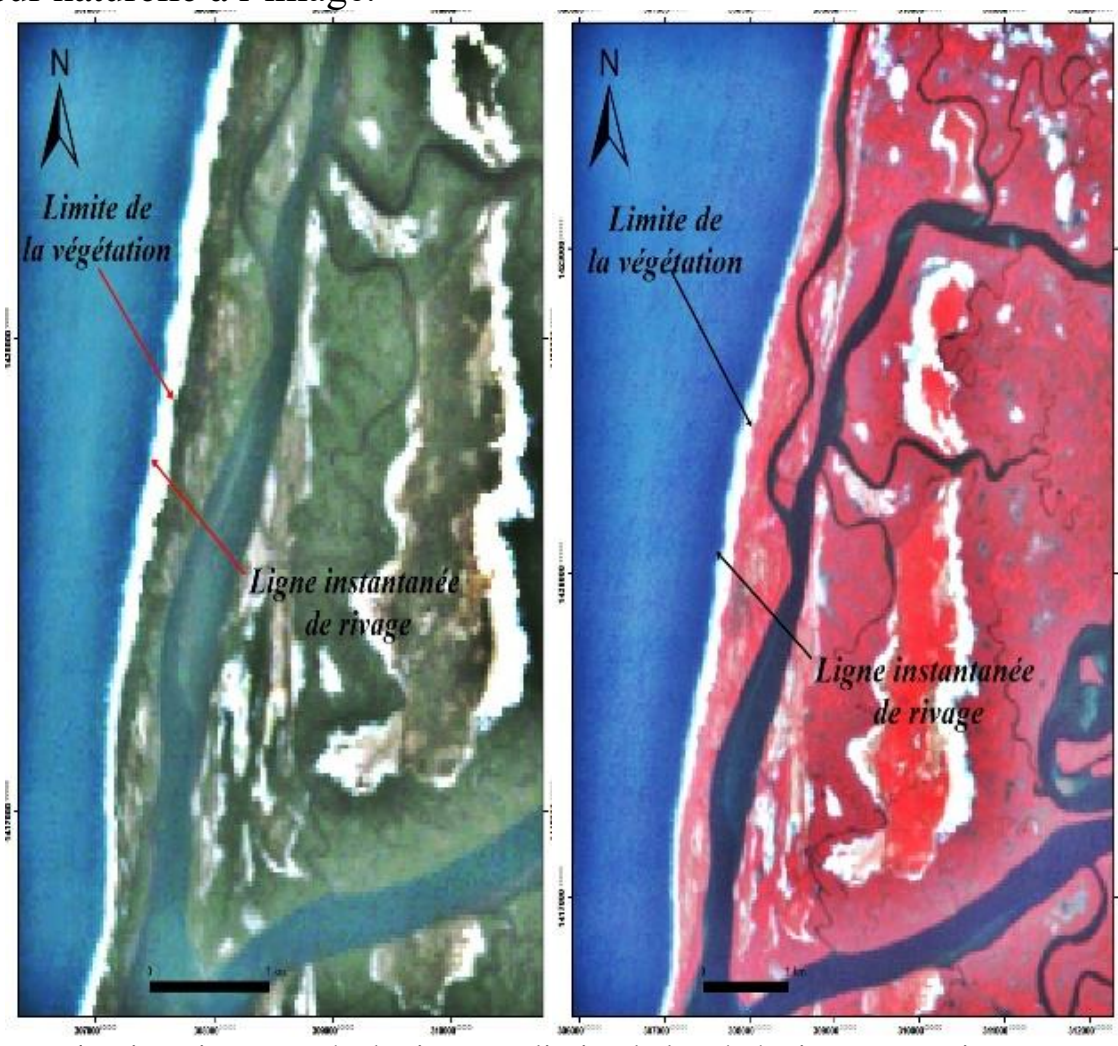

Figure 1: Ligne instantanée de rivage et limite de la végétation sur une image Landsat (composition colorée 3, 2, 1 à gauche et 4, 3, 2 à droite) sur un segment en Casamance

\subsection{Cartographie de l'évolution du trait de côte}

L'utilisation de l'imagerie spatiale pour la mesure de la cinématique du trait est pertinente dans le cas des littoraux extrêmement dynamiques dont l'évolution est supérieure à la marge d'erreur (Crowell et al., 1994; Fletcher et al., 2005; Faye, 2010). La représentation des résultats est peut se faire sur la base plusieurs échelles temporelles. Une échelle qui correspond à l'écart entre deux dates (deux traits de côte) et une qui prend en compte tous les traits de côte. Mais au préalable, il faut définir les indices de calcul de taux d'évolution.

L'End Point Rate (EPR) calcule le rapport de la distance entre le trait de côte correspondant à la date la plus ancienne et à la date la plus récente. Ainsi, lorsqu'on dispose uniquement de deux traits de côte, l'EPR reste un bon 
indice pour évaluer la cinématique (Moore, 2000; Thieler et al., 2005; Himmelstoss, 2009).

\subsubsection{Utilisation de l'outil « Digital Shoreline Analysis System» pour mesurer l'évolution du trait de côte}

Les méthodes statistiques d'extrapolation ou de calcul de tendance sont souvent utilisées en matière d'étude de la dynamique du trait de côte, à travers les outils d'analyse spatiale pour principalement anticiper sur l'érosion côtière (Emmanuel, 2017). C'est ainsi que nos travaux de numérisation ont été réalisés avec le logiciel ArcGIS 10.5®. A partir de là, les calculs statistiques des vitesses d'évolution du trait de côte sont effectués avec l'extension DSAS4.3. En effet, le « Digital Shoreline Analysis System » est une extension qui permet de faire des calculs sur les écarts des traits de côte déjà digitalisés à partir des images retenues. Développé depuis les années 1990 par l'US Geological Survey, DSAS fournit une méthode normalisée qu'on peut répéter pour le calcul de l'évolution statistique tout en analysant les changements passés, présents et futurs du littoral (Thieler et al., 2005).

La Digitalisation, analyse diachronique du trait de côte est basée sur la superposition de plusieurs images sur lesquelles peuvent respectivement être détectés les traits de côte en fonction des dates retenues. La première étape dans la digitalisation est d'abord la création de données cartographiques sur le trait de côte. Il s'agit de renseigner, après avoir créé un fichier de forme (Shp), le système de projection et le type de la donnée (linéaire).

Ainsi, les traits de côte peuvent être directement numérisés, puis exportées vers ArcGIS en passant le fichier $K M L$ en fichier SHP. En conséquence, le passage de ces données raster en vecteurs a permis d'obtenir des traits de côte sur les différentes images. C'est sur la base de ces traits de côte que les calculs de la vitesse d'évolution sont effectués à partir de l'outil «Digital Shoreline Analysis System.

\subsubsection{Calcul statistique avec DSAS}

Le calcul de la vitesse d'évolution du trait de côte nécessite préalablement la mise en place d'au moins deux traits de côte numérisés à différentes dates. Par conséquent, avec l'outil DSAS de ArcGIS, le calcul des taux d'évolution est automatisé suivant les manipulations exigées. Le principe général de cet outil est de mesurer les écarts entre les traits de côte d'une même série et de calculer les statistiques des taux d'évolution (en m/an). Pour cela, l'exploitation de l'outil demande au préalable une mise en forme rigoureuse des données dans une géodatabase personnalisée, la création d'une ligne de base et de transects équidistants, une estimation de l'incertitude liée à la méthode ainsi que le choix des statistiques de calculs des taux d'évolution (Juigner, 2012). Lorsque tous les paramètres d'entrée sont correctement 
renseignés, DSAS génère automatiquement, selon le pas de mesure défini, des transects perpendiculaires au linéaire côtier, mesure les écarts entre les traits de côte et calcule les taux moyens de déplacement le long de chaque transect (Faye et al., 2013; Himmelstoss et al., 2017; Thieler et al., 2005).

Les indices calculés, pour la représentation de l'intervalle de deux dates, l'EPR (End Point Rate) est l'indice le plus pertinent. Concernant le taux d'évolution globale de toute la série, l'indice Low Regression Rate-of-change (LRR) est le plus pertinent. En effet, ces deux indices ont chacun une pertinence en fonction des informations à représenter.

L'EPR permet de mesurer l'écart entre deux traits de côte successifs. Il s'agit du rapport de la distance entre le trait de côte correspondant à la date la plus ancienne et à la date la plus récente. Ainsi, lorsqu'on dispose uniquement de deux traits de côte, l'EPR reste un bon indice pour évaluer la cinématique (Thieler et al., 2005 ; Himmelstoss et al., 2017).

Le LRR, lui, permet d'estimer l'évolution de chaque segment sur l'ensemble de la période considérée. Le nombre d'individus de la série statistique est celui du nombre de traits de côte. Ainsi, si ce nombre est faible comme pour l'EPR, cela pose un problème de pertinence et de robustesse des résultats. Par conséquent, calculant le taux d'évolution annuelle de la ligne de référence le long de chaque transect, l'EPR est donc le plus pertinent lorsqu'on analyse la cinématique littorale pour plus de deux dates, car la méthode de calcul tient compte de la distance évolutive du trait de côte sur toute la période considérée (Faye et al., 2013; Thior et al., 2021).

Les opérations de pré-calculs, concerne la création tout au départ une géodatabase personnalisée composée essentiellement de deux entités: d'abord, une première entité contenant une ou plusieurs lignes de références nommées baseline, ensuite, une seconde entité constituée de traits de côte déjà numérisés sur ArcMap nommée shorelines. C'est pour cela que la zone de tampon ou buffer_shoreline permet de stabiliser l'espace linéaire dans lequel les traits de côte ont été numérisés (fig. 2). 


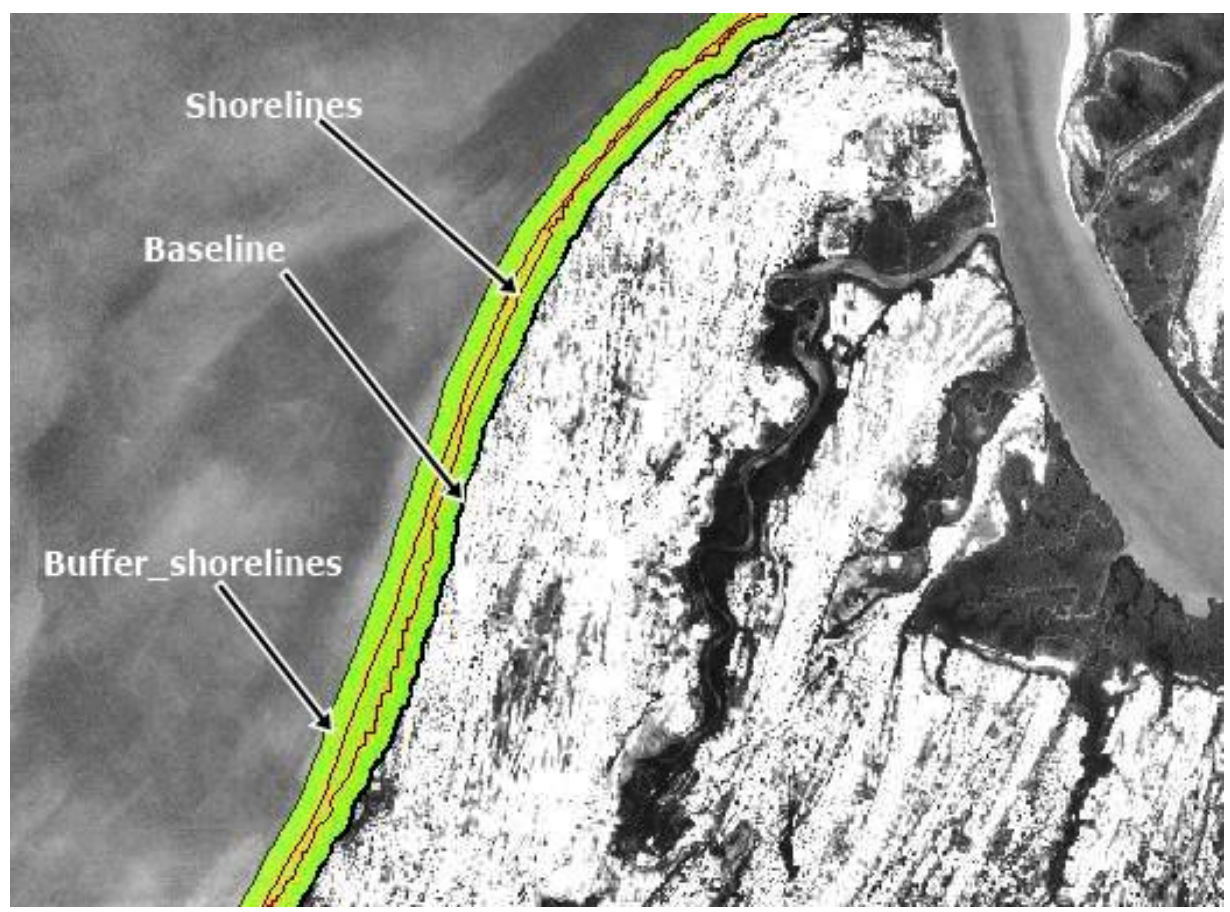

Figure 2: Les entités d'une géodabase personnalisée (Thior et al., 2019)

La baseline permet de numériser une ligne de base imaginaire à partir de laquelle DSAS crée des transects qui coupent les différents traits de côte. C'est donc à partir de cette Baseline que tous les transects ont pris repère en étant perpendiculaires à celle-ci qui, elle aussi, doit être parallèle à la côte. Cependant, il arrive que les transects soient déformés du fait de l'irrégularité de la côte. Ce qui entraîne parfois des transects aberrants ou qui s'entrecoupent avant leur intersection sur les traits de côte. Il est ainsi possible de les corriger en les redressant (pour les transects qui s'entrecoupent) ou de supprimer les transects aberrants.

Les shorelines représentent les traits de côte déjà numérisés dans la géodatabase. Il s'agit, en effet, des traits de côte auxquels la baseline doit être parallèle et devant servir de mesure dans l'environnement de DSAS. Ainsi, pour pouvoir effectuer un calcul d'indices (EPR, LRR), les shorelines doivent être au minimum deux entités de dates ou encore d'heures différentes.

Les Buffer-Shoreline permettent d'effectuer un calcul sur DSAS, il faut circonscrire le segment sur lequel les mesures de variation de l'évolution du trait de côte seront réalisées. C'est en effet la zone de tampon qui permet de définir de quel côté de la baseline seront tracés les profils.

En effet, la zone de tampon donne deux possibilités de position de la baseline (du côté de la mer ou du côté de la terre) et cela, en fonction du sens de la baseline, définie par les sommets de départ et d'arrivée. Mais, dans cette 
démarche, toutes les baseline ont été numérisées à partir de la limite du côté terrestre de la zone tampon, avec une distance de $150 \mathrm{~m}$ la séparant des shorelines.

Les calculs statistiques sont des opérations de pré-calcul qui sont en réalité une configuration de l'environnement de DSAS et qui précèdent l'étape de calcul statistique. Cette étape s'est faite d'abord par une création de transects, ensuite par un calcul des indices, puis la représentation graphique des statistiques issues des tables attributaires des indices calculés selon leur pertinence.

La mise en place de transects qui sont des profils perpendiculaires à la ligne de base (baseline) qui permettent de mesurer la variation des taux d'évolution des traits de côte. Dans cette démarche, le nombre de transects est égal au nombre de profils utilisés et dépend de la longueur du segment (site) étudié. L'équidistance entre les profils mesurés automatiquement par DSAS est estimée en (m). Cependant, il arrive très souvent, sur la frange de certains littoraux, que certains transects apparaissent de manière aberrante, suite à l'existence d'une ouverture d'embouchure ${ }^{3}$ au niveau du segment étudié. Or, les transects doivent couper tous les traits de côte qui leur sont perpendiculaires. D'autres sources de transects aberrants peuvent émaner de la sinuosité du linéaire côtier pouvant entraîner des saccades avant même leur intersection sur le shorelines. Ainsi, malgré les paramètres choisis, une partie d'entre eux risquent de venir fausser l'information à cause de leur positionnement et de la nature du linéaire côtier (Himmelstoss et al., 2009). Il convient nécessairement de les corriger ou de les supprimer pour éviter les mesures inexistantes ou nulles. Par conséquent, après la configuration en fonction des besoins, DSAS génère automatiquement la position des transects (profils) en vue d'effectuer les calculs d'indices.

\subsubsection{Calcul des indices}

Le calcul statistique consiste, en fonction des indices considérés, à faire ressortir les données tabulaires calculées automatiquement par DSAS. En effet, c'est l'analyse automatique de tous les transects qui permet de définir des secteurs d'évolution type et d'en calculer les valeurs moyennes (Morton et al., 2004 ; Juigner, 2012 ; Faye et al., 2013). Ainsi, après calcul, on obtient dans la table attributaire des transects les différents indices pris en compte lors de la configuration et dont les plus pertinents dans cette étude sont les End Point Rate (EPR) et les Linear Regression Rate-of-change (LRR).

\footnotetext{
${ }^{3} \mathrm{Au}$ niveau des embouchures il n'est pas possible de tracer un trait de côte entre les rives du fait de la rupture de la plage. Mais la baseline la prend en compte et crée à ce niveau des transects de fausses mesures
} 
La représentation graphique est conditionnée par les résultats statistiques des indices calculés. Elle restitue visuellement les évolutions mesurées sous plusieurs formes de graphiques. C'est pourquoi, en fonction des indices, deux types de représentation graphique sont utilisés dans cette étude. Une représentation basée sur la classification des transects sur Arc GIS®, permettant de voir le taux d'évolution entre deux traits de côte.

Une deuxième représentation graphique sous forme d'aire ou au niveau de l'axe des ordonnées est réalisée. On a l'axe longitudinal de l'étendue linéaire de la plage et l'axe des abscisses qui permet de voir proportionnellement l'évolution du trait de côte. Cette représentation n'est pas toujours applicable naturellement sur toutes les côtes. En conséquence, cette organisation graphique paraît en effet naturelle lorsque le trait de côte étudié est orienté Nord-Sud sur les littoraux atlantiques. Mais, il se peut que, par endroits, la côte ait une orientation Ouest-Est. Dans ce cas de figure, il est plus représentatif de réorienter le graphique des aires de sorte à avoir une vue qui facilite l'observation (Faye et al., 2013).

Ces représentations réalisées à partir de l'interface graphique de ArGis permettent de voir une situation globale de l'évolution de la plage par secteur. En revanche, ces secteurs, constituant l'échelle d'analyse élémentaire pour la partie statistique et graphique, transposent une discrimination du rivage permettant de rendre compte des variations spatiales de la cinématique du littoral (Byrnes, 1994 ; Martinez et al., 2018).

\subsubsection{Les marges d'erreur}

La précision des résultats des analyses du trait de côte est très souvent influencée par plusieurs sources d'erreurs surtout lorsque la position du trait de côte est historique (Crowell et al., 1994; Moore, 2000; Robin et Gourmelon, 2016 ; Juigner, 2012 ; Moussaid et al., 2015 ; Esmail et al., 2019). La qualité des résultats d'une analyse diachronique est donc intimement liée à la connaissance de la marge d'erreur (Juigner, 2012). En effet, les marges d'erreur permettent de caractériser la situation (érosion, stabilité, accrétion) sur une période bien déterminée. L'estimation de ces risques d'erreurs doit conduire à l'élaboration d'une marge d'erreur, qui sera prise en compte dans l'interprétation des résultats (Durand, 2000). C'est la raison pour laquelle les erreurs les plus récurrentes en cinématique du littoral, particulièrement en ce qui concerne l'analyse du trait de côte, sont principalement les erreurs de positionnement et les erreurs techniques.

Les erreurs techniques désignent les marges d'erreur liées à la nature de l'image sont un souci pour la fiabilité des résultats escomptés. En réalité, la plupart des études, qui s'intéressent à la variabilité du trait de côte, à l'aide d'un SIG, se sont réalisées à l'aide d'imageries satellitaires ou encore de photographies aériennes. La superposition de deux images de nature différente 
nécessite généralement une rigueur dans la correction. Trepanier et al., (2010) ont montré que les corrections géométriques rigoureuses sont un exercice d'harmonisation des capteurs, visant à stabiliser la dynamique des images $\mathrm{HRV}$, de façon à simuler l'enregistrement de deux images par un même capteur, où l'on peut être en mesure d'effectuer une bonne comparaison des images. Or, sur une image Landsat, par exemple, lorsque sa résolution spatiale est de $30 \mathrm{~m}$, les objets au sol, n'ayant pas une taille supérieure à la résolution spatiale, ne vont plus exister.

Pourtant, ces objets existent bien sur une photographie aérienne de 1 $\mathrm{m}$ de résolution. Selon Kuling (2016), la structure en pixels carrés de l'imagerie induit donc une simplification de la réalité. La dimension du pixel varie selon les systèmes d'acquisition des données, et lorsqu'une photographie ou tout autre document est numérisé(e), la dimension du pixel est fonction de l'échelle de la photographie et de la résolution du scanner. Ainsi, pour un objet quelconque, ceci implique globalement un décalage plus ou moins fort entre ses coordonnées dans le SIG, défini à partir de l'imagerie et ses coordonnées réelles. Ce type d'erreur appelé erreur pixel laisse voir au moins une légère différence entre la position réelle et la position spatiale des traits de côte numérisés, d'où l'importance d'un bon géoréférencement qui peut permettre d'avoir le plus faible RMS (Root Mean Square) exprimé en Pixel.

Les autres erreurs techniques peuvent émaner de la digitalisation. En effet, lors de la numérisation du linéaire côtier, il arrive que l'opérateur manque de précision dans la visualisation de la ligne de référence, surtout quand le support et l'écran présentent des défaillances. Il est donc beaucoup plus prudent de répéter l'opération de digitalisation du même trait de côte et voir les écarts entre eux pour pouvoir définir les erreurs (Juigner, 2012 ; Himmelstoss et al., 2009 ; Fletcher et al., 2005 ; Faye et al,. 2013).

Les erreurs météo-marines renvoient aux marges d'erreur liées à la variabilité du marqueur du trait de côte, elles sont essentiellement liées aux conditions météo-marines, c'est-à-dire la variation de la marée. Ces erreurs sont prises en compte lorsque les lignes de référence considérées sont, soit une limite d'humectation, soit une ligne instantanée de rivage. En outre, sur les côtes à grande fréquence de tempêtes, les erreurs liées aux conditions météomarines doivent également être prises en compte. Selon Juigner (2012), l'intégration de ces erreurs dans la marge globale est d'autant plus importante lorsque les taux d'évolution sont reportés pour établir un trait de côte prédit via une régression linéaire. Cette démarche s'explique par le fait que le trait de côte, extrait à partir de photos aériennes, traduit une position à un instant donné (T) sans tenir compte de la dynamique ( $\mathrm{T}-1$ à $\mathrm{T}+1)$ dans laquelle elle évolue. Le but est d'obtenir un intervalle de position dans lequel se situe le vrai trait de côte dont la valeur résume davantage la dynamique de $\mathrm{T}-1$ à $\mathrm{T}+1$. 
L'idéal en cinématique du littoral est de disposer d'une série d'images ayant des conditions météo-marines identiques. Cependant, il est très rare de tomber sur un document historique où ces informations sont mentionnées. C'est pourquoi il est plus prudent de travailler avec des indicateurs de trait de côte indépendants du type de marée, lorsque les côtes sur lesquelles porte l'analyse spatiale présentent des lignes de référence physiques. ${ }^{4}$

A côté de ces difficultés sur les lignes de référence, interviennent des erreurs qui peuvent être liées au type de projection, à la méthode de reprographie et à l'échelle utilisée (Boak et Turner, 2005). Il est donc essentiel de noter que dans la littérature existante en analyse spatiale du trait de côte, il existe plusieurs démarches mises en place dont les résultats tournent autour d'un objet commun qui est la cinématique du littoral. Toutefois, les approches dépendent en premier lieu du type de côte (rocheuse/sableuse; microtidale/macrotidale; mobile/fixée ; végétalisée ou non, etc.), d'où découle le choix d'une ligne de référence ou de plusieurs. S'ensuit la diversité des supports et des techniques d'extraction dont dépend la marge d'erreur (Juigner, 2017 ; Durand, 2000 ; Crowell et al., 1994).

Intégration de la marge d'erreur intègrent les résultats de mesure des taux d'évolution du trait de côte, quelle que soit l'approche par analyse spatiale souffrent de plusieurs sources d'erreurs. (Crowell et al., 1994 ; Moore, 2000 ; Robin et Gourmelon, 2016 ; Juigner, 2012, Sadio, 2017; Thior et al., 2019; Thior et al., 2021). La qualité des résultats d'une analyse diachronique est donc intimement liée à la connaissance de la marge d'erreur (Juigner, 2017). En effet, les marges d'erreur permettent de caractériser la situation (érosion, stabilité, accrétion) sur une période bien déterminée. L'estimation de ces risques d'erreurs doit conduire à l'élaboration d'une marge d'erreur, qui sera prise en compte dans l'interprétation des résultats (Durand, 2000 ; Gibbs et al., 2017 : Gould et al., 2015). C'est la raison pour laquelle, les erreurs les plus récurrentes en cinématique du littoral, particulièrement en ce qui concerne l'analyse du trait de côte, sont principalement les erreurs de positionnement et les erreurs techniques.

L'erreur globale, appelée Erreur de Position Globale du trait de côte, est calculée en prenant la racine carrée de la somme des carrées de chaque erreur : erreur de pixel, de géoréférencement, de digitalisation, oscillation (Juigner, 2012 ; Fletcher et al., 2005; Thior et al., 2021).

Dans cette étude, trois sources d'erreur sont prises en compte :

- l'erreur de géoréférencement (RMS Root Mean Square Error). Lors du géoréférencement, le logiciel (ArcGIS) calcule automatiquement l'erreur pour effectuer la correction géométrique ;

${ }^{4}$ Les limites géomorphologiques ou les limites de la végétation 
- l'erreur liée à la variation de la marée est obtenue par le rapport de la hauteur de la marée sur la pente moyenne de la plage

- l'erreur liée à la digitalisation fait partie des erreurs aléatoires qu'il faut intégrer dans l'analyse des résultats. Mais il n'existe pas encore à ce jour une méthode objective pour l'évaluer (Morton et al., 2004 ; Thieler et al., 2005). Néanmoins, il est recommandé de l'estimer en effectuant la numérisation plusieurs fois. L'erreur émanant de la numérisation de chaque ligne de rivage étant obtenue par addition de la moyenne des écarts à 2 écarts-types de ces écarts et à la taille du pixel (Moore et Gibbs 2017 ; Fletcher et al., 2005 ; Faye, 2010 ; Sadio, 2017 ) La marge d'erreur émanant de la numérisation est obtenue en cumulant sa moyenne +2 écarts-types + taille pixel.

Ainsi, on calcule pour chaque année la valeur des trois erreurs déjà identifiées sur les images. Cela donne donc les équations suivantes :

$$
\begin{array}{cc}
\boldsymbol{E p t}=\sqrt{+E g^{2}+} & \begin{array}{c}
\text { Ept: Erreur de position globale du trait de côte } \\
\text { Eg : Erreur de Géoréférencement (RMS) } \\
\text { Ed : Erreur de digitalisation }
\end{array} \\
\boldsymbol{E} \boldsymbol{\alpha}=\frac{\sqrt{E p t 1^{2}+E p t 2^{2}}}{\text { Eóriode }: \text { Erreur de l'oscillation de marée }} \\
\text { E } \alpha: \text { Erreur globale en moyenne en } \mathrm{m} / \mathrm{an}
\end{array}
$$

\section{Conclusion}

Les mesures actuelles et les méthodes de calcul des taux de variation varient d'une étude à l'autre; ce qui exclut la combinaison des résultats de l'étude dans les évaluations nationales ou régionales. Mais, en cinématique du littoral, le traitement numérique de l'information géographique est presque devenu une démarche standard bien que des améliorations de la technologie d'analyse spatio-temporelle soient en perpétuelle avancée. L'application de cette démarche suppose d'abord que l'on dispose de documents cartographiques anciens, les premières devant être les plus vieilles parmi celles dont l'exploitation est aisée. L'utilisation des documents cartographiques anciens est aussi d'une grande importance à la contribution de la connaissance de la position initiale du trait de côte surtout quand les outils de la géomatique sont appliqués. Cependant, quelque soit la qualité des données géospatiales utilisées, les résultats issus de leur surperposition souffrent d'erreurs qu'il faut intégrer dans l'analyse.

\section{References:}

1. Barry M., (2016). Erosion côtière et impacts dans la commune de Kafountine (Basse Casamance). Mémoire de Master, UAS/Z, 121 p. 
2. Bird E.C.F., (2007) Coastal geomorphology. An introduction. John WILEY and Sons Ltd, Chichester, 411 p..

3. Boak, E.H., Turner, I.L., (2005). Shoreline Definition and Detection: A Review. Journal of Coastal Research, 21 (4), 688-703. West Palm Beach (Florida), ISSN 0749-0208.

4. Bourles B., (1998). Variabilité de la hauteur dynamique dans l'Atlantique Tropical vue par altimétrie. In Évolution des littoraux de la Guyane et de la Zone Caraïbe Méridionale pendant le Quaternaire. PICG 274/ORSTOM. Coll. Colloques et Séminaires, IRD. Paris.

5. Byrnes M., (1994). Shoreline position and nearshore bathymetric change, US Army Corps of Engineers. Waterways Experiment Station. VCSBURG. Report, CERC-94-9, p.61-143.

6. Crowell M., Leatherman S., Buckley M.K., (1994). Shoreline change rate analysis: long term versus short term data. Shore and Beach, vol. $61, \mathrm{n}^{\circ} 2, \mathrm{p} .13-20$.

7. Cuq F., Campredon P., Giraudet J., Giraudet E., Gourmelon F., Pennober G., Da silva A. S., 2001 :Un Système d'Information Géographique pour l'aide à la gestion intégrée de l'Archipel des Bijagos (Guinée-Bissau), CNRS/UICN, Carte (feuilles à 1/50 000) et notice, $88 \mathrm{p}$.

8. Diaw A.T., 2003 : L'état de la géomatique au Sénégal. Annales de la Faculté des Lettres et Sciences humaines, n³0, p. 49 - 63.

9. Diop S., (1900) La Côte ouest-africaine, du Saloum (Sénégal) à la Mellacorée (République de Guinée). Paris : ORSTOM, 1990, 366 p.

10. Djagouna ERIC M. Valere., (2016). Cartographie De La Dynamique Du Trait De Côte A Grand-Lahou : Utilisation De L'outil «Digital ShorelineAnalysis,System(Dsas)»doi:10.19044/esj.2016.v12n36p327 URL:http://dx.doi.org/10.19044/esj.2016.v12n36p327.

11. Dolan R., Hayden B. P., Heywood J., 1978: A new photogrammetric method for determining shoreline erosion. Coastal Engineering, vol. 2, pp. 21 - 39.

12. Durand P., (2004) L'évolution des plages de l'Ouest du golfe du Lion au XXe siècle - Cinématique du trait de côte, dynamique sédimentaire, analyse prévisionnelle. Thèse de Doctorat de l'Université de Lyon, $321 \mathrm{p}$.

13. Emmanuel B., (2017). Etude des dynamiques du trait de côte de la région Bretagne à différentes échelles spatio-temporelles. Géographie. Université de Bretagne Occidentale - Brest. Français. <NNT : 2017BRES0010>

14. Esmail M., Mahmod W.E., and Fatha H., (2019) Assessment and prediction of shoreline change using multi-temporal satellite images 
and statistics: Case study of Damietta coast, Egyp. Ocean research $\mathrm{n}^{\circ} 82,274-282$.

15. Faye I,. Giraudet E,. Gourmelon F,. Henaff A (2013) : Cartographie normalisée du trait de côte Mappemonde. 12p.

16. Faye I., (2010). Dynamique du trait de côte sur les littoraux sableux de la Mauritanie à la Guinée-Bissau (Afrique de l'ouest) : approche régionale et locale par photo-interprétation, traitement d'images et analyses de cartes anciennes. Thèse de l'université de Bretagne occidentale, $321 \mathrm{p}$.

17. Fletcher C.H., Rooney J.J., Barbee M., LIM S.C., and Richmond B., (2005) Mapping shoreline change using digital orthophotogrammetry on Maui, Hawaii. Journal of Coastal, Research, Special Issue n ${ }^{\circ} 38$, pp $106-124$.

18. Gibbs, A.E., Ohman, K.A., Coppersmith, R., and Richmond, B.M., 2017: National Assessment of Shoreline Change: A GIS compilation of updated vector shorelines and associated shoreline change data for the north coast of Alaska, U.S. Canadian border to Icy Cape: U.S. Geological Survey data release, https://doi.org/10.5066/F72Z13N1.

19. Girard M.C., 2010 : Traitement des données de télédétection: Environnement et ressources naturelles. 2ème édition, Dunod, $553 \mathrm{p}$.

20. Gould A I., Kinsman N.E.M., Hendricks M.D., 2015: Guide to Projected Shoreline Positions in the Alaska Shoreline Change Tool, (), Department of Natural Resources, Division of Geological \& Geophysical Surveys. Miscellaneous Publication 158, 11 p.

21. Hapke C.J., Nathaniel G., Plant E. Henderson W C., Schwab T.R.N., 2016 Decoupling processes and scales of shoreline morphodynamics, Marine Geology, Volume 381, 2016, Pages 42-53, ISSN 0025 3227,https://doi.org/10.1016/j.margeo.2016.08.008.

22. Himmelstoss, E.A., (2009). 'DSAS 4.0 Installation Instructions andUser Guide' in: Thieler, E.R., Himmelstoss, E.A., Zichichi, J.L., and Ergul, Ayhan. 2009 Digital Shoreline Analysis System (DSAS) version 4.0 An ArcGIS extension for calculating shoreline change: 130 U.S. Geological Survey Open-File Report 2008-1278. Updated forversion 4.3.

23. Juigner M., (2012). Cinématique d'un trait de côte sableux en Vendée entre 1920 et 2010, Méthode et analyse, Géolittomer CNRSUMR 6554 LETG, Université de Nantes, 13p.

24. Kuling B., 2016 : Télédétection en milieu littoral, variation du trait de côte avec DSAS 4.3, partenariat public / privé: USGS (BRGM étatsunien) + Innovate! Inc 125p.

25. Le Berre I., Henaff A., Devogele T., MascreT A., et Wenzel F., (2008). «SPOT5 : un outil pertinent pour le suivi du trait de côte ?», Norois 
[En ligne], 196 | 2005/3, mis en ligne le 15 décembre 2008, consulté le 30 septembre $2016 \mathrm{http} / / /$ norois.revues.org/378 ; DOI : 10.4000/norois. 378 .

26. Martínez C., López M C., Winckler P., Hidalgo H., Godoy E., Agredano R., 2018: Coastal erosion in Central Chile: A new hazard?, Ocean \& Coastal Management, Volume156,Pages 141155,ISSN0964-

5691,https://doi.org/10.1016/j.ocecoaman.2017.07.011. (http://www.sciencedirect.com/science/article/pii/S096456911730156 4)

27. Moore 1. J., (2000). Shoreline mapping techniques. Journal of Coastal Research, vol. 16, $n^{\circ} 1, p .111-124$.

28. Morton. R. A., Miller, T. L., Moore, L. J., 2004: National assessment of shoreline change: Part 1: Historical shoreline changes and associated coastal land loss along the U.S. Gulf of Mexico: U.S. Geological Survey Open-file Report 2004-1043, 45p.

29. Moussaid J.,Fora A.A.,Zourarah B.,Maanan M and Maanan M., 2015:Using automatic computation to analyze the rate of shoreline change on the Kenitra coast, Morocco.Ocean Engineering $\mathrm{n}^{\circ} 102,71$ 77.

30. Pennober G., 1999 : Analyse spatiale de l'environnement Côtier de l'Archipel des Bijagos (Guinée Bissau), Thèse de Doctorat, Université de Bretagne Occidentale, 232p.

31. Robin M., Gourmelon F., (2016). «La télédétection et les SIG dans les espaces côtiers. Eléments de synthèse à travers le parcours de François Cuq », Norois [En ligne], 196 | 2005/3, mis en ligne le 15 décembre 2008, consulté le 12 août 2016. URL : http://norois.revues.org/368 ; DOI : 10.4000/norois.368.

32. Stafford D.B., Langfelder J. (1971) Air photo survey of coastal erosion. Photogrammetric Engineering, vol. 37, p. 565 - 575.

33. Thieler, E. R., Martin, D., AND Ergul, A., (2005). The Digital Shoreline Analysis System, version 2.0: Shoreline change measurement software extension for ArcView: USGS U.S. Geological Survey Open-File Report 03-076.

34. Thior M., Sané T., Dièye El H Balla Sy O., Cissokho D., Ba B D., Descroix L., (2019): " Coastline dynamics of the northern Lower Casamance (Senegal) andsouthern Gambia littoral from 1968 to 2017» Journal of African Earth Sciences 160 (2019)103611 1464-343X/ (C) 2019 Elsevier Ltd.8p.

35. Thior M., Sy A A., Cissé I., Dièye El H Balla Sané T., Ba B D., Solly B., Descroix L., (2021): «Approche cartographique de l'évolution du trait de côte dans l'estuaire de la Casamance » Mappemonde, $\mathrm{N}^{\circ} 131$, 
2021.

ISSN

1769-7298,

URL

https://journals.openedition.org/mappemonde/5939.

36. Trepanier I J., Dubois M. M., Bonn F., (2010) Suivi de l'évolution du trait de côte à partir d'images HRV (XS) de SPOT: Application au delta du fleuve Rouge, Viêt-Nam, International Journal of Remote Sensing, 23:5,917-937, DOI: 10.1080/01431160110070348 